\title{
Titania-doped tantala/silica coatings for gravitational-wave detection
}

\author{
Gregory M Harry ${ }^{1}$, Matthew R Abernathy ${ }^{1}$, Andres E Becerra-Toledo ${ }^{1}$, \\ Helena Armandula ${ }^{2}$, Eric Black ${ }^{2}$, Kate Dooley ${ }^{2}$, Matt Eichenfield ${ }^{2}$, \\ Chinyere Nwabugwu ${ }^{2}$, Akira Villar ${ }^{2}$, D R M Crooks ${ }^{3}$, \\ Gianpietro Cagnoli ${ }^{3}$, Jim Hough ${ }^{3}$, Colin R How ${ }^{3}$, Ian MacLaren ${ }^{3}$, \\ Peter Murray $^{3}$, Stuart Reid ${ }^{3}$, Sheila Rowan ${ }^{3}$, Peter H Sneddon ${ }^{3}$, \\ Martin M Fejer ${ }^{4}$, Roger Route ${ }^{4}$, Steven D Penn ${ }^{5}$, Patrick Ganau ${ }^{6}$, \\ Jean-Marie Mackowski ${ }^{6}$, Christophe Michel ${ }^{6}$, Laurent Pinard ${ }^{6}$ and \\ Alban Remillieux ${ }^{6}$ \\ ${ }^{1}$ LIGO Laboratory, Massachusetts Institute of Technology, NW17-161, Cambridge, MA 01239, \\ USA \\ ${ }^{2}$ LIGO Laboratory, California Institute of Technology, Pasadena, CA 91125, USA \\ ${ }^{3}$ Department of Physics and Astronomy, The University of Glasgow, Glasgow, G12 8QQ, UK \\ ${ }^{4}$ Edward L Ginzton Laboratory, Stanford University, Stanford, CA 94305, USA \\ 5 Physics Department, Hobart and William Smith Colleges, Geneva, NY 14456 \\ ${ }^{6}$ Laboratoire Matérial Advancée-Virgo, Lyon, France \\ E-mail: gharry@ligo.mit.edu
}

Received 21 September 2006, in final form 20 November 2006

Published 8 December 2006

Online at stacks.iop.org/CQG/24/405

\begin{abstract}
Reducing thermal noise from optical coatings is crucial to reaching the required sensitivity in next generation interferometric gravitational-wave detectors. Here we show that adding $\mathrm{TiO}_{2}$ to $\mathrm{Ta}_{2} \mathrm{O}_{5}$ in $\mathrm{Ta}_{2} \mathrm{O}_{5} / \mathrm{SiO}_{2}$ coatings reduces the internal friction and in addition present data confirming it reduces thermal noise. We also show that $\mathrm{TiO}_{2}$-doped $\mathrm{Ta}_{2} \mathrm{O}_{5} / \mathrm{SiO}_{2}$ coatings are close to satisfying the optical absorption requirements of second generation gravitational-wave detectors.
\end{abstract}

PACS numbers: $04.80 . \mathrm{Nn}, 62.40 .+\mathrm{i}, 95.55 . \mathrm{Ym}$

(Some figures in this article are in colour only in the electronic version)

\section{Introduction}

Interferometric gravitational-wave detectors are now operating in the United States [1], Europe $[1,2]$ and Japan [3]. Second generation detectors [4] are being designed which will have the sensitivity to make likely an actual detection of a gravitational wave [5]. This will 
require reducing all noise sources, but especially the thermal noise which is predicted to be the limiting noise in the most sensitive band around $100 \mathrm{~Hz}$. Much of this thermal noise will come from the optical coatings of the interferometer mirrors [6]. Coatings with improved thermal noise performance will allow for greater sensitivity to gravitational waves and improved astrophysical performance. Coating thermal noise is also the limiting noise source for laser frequency stabilization [7], making this research effort important for other precision experiments.

Thermal noise is caused by mechanical loss in the system in accordance with the fluctuation-dissipation theorem [8]. Directly applying this theorem to the case of a Gaussianprofile laser sensing, the position of a coated mirror yields [9]

$$
S_{x}(f)=2 k_{\mathrm{B}} T \phi_{\mathrm{eff}}\left(1-\sigma^{2}\right) /\left(\pi^{3 / 2} f w Y\right),
$$

for the thermal noise. Here $S_{x}(f)$ is the power spectral density of position noise, $k_{\mathrm{B}}$ is Boltzmann's constant, $T$ is the temperature, $\sigma$ is the Poisson ratio of the substrate material, $w$ is the half-width of the Gaussian laser beam and $\phi_{\mathrm{eff}}$ is the effective loss angle of the mirror. The loss angle can be written as [11]

$$
\begin{aligned}
\phi_{\mathrm{eff}}=\phi+d / & \left(\sqrt{\pi} w Y_{\perp}\right)\left(\left(Y /\left(1-\sigma^{2}\right)-2 \sigma_{\perp}^{2} Y Y_{\|} /\left(Y_{\perp}\left(1-\sigma^{2}\right)\left(1-\sigma_{\|}\right)\right)\right) \phi_{\perp}\right. \\
& +Y_{\|} \sigma_{\perp}(1-2 \sigma) /\left(\left(1-\sigma_{\|}\right)(1-\sigma)\right)\left(\phi_{\|}-\phi_{\perp}\right) \\
& \left.+Y_{\|} Y_{\perp}(1+\sigma)(1-2 \sigma)^{2} /\left(Y\left(1-\sigma_{\|}^{2}\right)(1-\sigma)\right) \phi_{\|}\right),
\end{aligned}
$$

where $d$ is the coating thickness, $Y, \sigma$, and $\phi$ are Young's moduli, Poisson's ratios and loss angles of the substrate (no subscript), and for the coating for stresses perpendicular $(\perp)$ and parallel $\left(_{\|}\right)$to the optic face.

In addition to low thermal noise, the coatings must also satisfy strict thermal and optical requirements. The Fabry-Perot cavities that make up the arms of these detectors must have high finesse, limiting the acceptable transmission and scatter to a few parts per million (ppm). In addition, the transmission must be matched between mirrors to better than $1 \%$ so that the two arms will have nearly equal finesse. The absorption has a stricter requirement, better than $0.5 \mathrm{ppm}$, due to thermal lensing considerations [10]. Thermal considerations also dictate that the absorption be as uniform as possible across the face of the optic.

\section{Background}

Study of multilayer dielectric optical coatings is an ongoing research project in the gravitational-wave detection community $[6,11-20]$. The coating used in initial interferometers, alternating $\lambda / 4$ layers of $\mathrm{SiO}_{2}$ and $\mathrm{Ta}_{2} \mathrm{O}_{5}$, was studied to determine if the mechanical loss was enough to cause thermal noise problems $[6,15]$. The particular coatings measured were coated by Research-Electro Optics (REO) of Boulder CO, USA. When it was determined that the loss was enough to cause limiting noise in next generation gravitationalwave detectors, research was carried out to determine the source of the mechanical loss in $\mathrm{SiO}_{2} / \mathrm{Ta}_{2} \mathrm{O}_{5}$ coatings [16]. This was done in collaboration with LMA/Virgo of Lyon, France, who coated the samples. This established that the loss came from internal friction in the coating materials rather than any interface effects between layers or between the substrate and the coating. It was also found that $\mathrm{Ta}_{2} \mathrm{O}_{5}$, rather than $\mathrm{SiO}_{2}$, was the dominant contributor to the coating mechanical loss. The loss angles of $\mathrm{SiO}_{2}$ and $\mathrm{Ta}_{2} \mathrm{O}_{5}$ were found to be [17]

$$
\phi_{\mathrm{SiO} 2}=(1.0 \pm 0.2) \times 10^{-4}+f(1.1 \pm 0.5) \times 10^{-9},
$$




$$
\phi_{\mathrm{Ta} 205}=(3.8 \pm 0.2) \times 10^{-4}+f(1.8 \pm 0.5) \times 10^{-9},
$$

as a function of frequency $f$.

The thermal noise from optical coatings has also been directly observed in two small scale interferometers, one at Caltech [18] and one in Japan [21]. The Caltech measurement was on an REO coating, and found thermal noise at a few kilohertz to be consistent with

$$
\phi_{\text {eff }}=6.5 \pm 0.4 \times 10^{-6},
$$

where $\phi_{\mathrm{eff}}$ is from equation (2). The values determined from the modal Q measurements in equations (3) and (4) predict

$$
\phi_{\text {eff }}=6.4 \pm 0.3 \times 10^{-6} .
$$

Optical absorption was also measured for $\mathrm{Ta}_{2} \mathrm{O}_{5} / \mathrm{SiO}_{2}$ coatings. Coatings from $\mathrm{REO}$ and LMA/Virgo gave similar results [22],

$$
\begin{aligned}
& \alpha_{\mathrm{REO}}=0.3 \pm 0.1 \mathrm{ppm} \\
& \alpha_{\mathrm{LMA}}=0.4 \pm 0.1 \mathrm{ppm} .
\end{aligned}
$$

\section{Measurement}

The next stage of coating research has been to improve the mechanical loss without significantly degrading the optical absorption. Adding $\mathrm{TiO}_{2}$ as a dopant to $\mathrm{Ta}_{2} \mathrm{O}_{5}$ was tried because it has a high Young's modulus, its atomic size allows for dense packing in the Ta and $\mathrm{O}$ matrix and the melting point of the $\mathrm{TiO}_{2} / \mathrm{Ta}_{2} \mathrm{O}_{5}$ alloy is relatively high, indicating a stable amorphous structure.

Silica substrates were coated with the $\mathrm{TiO}_{2}$-doped $\mathrm{Ta}_{2} \mathrm{O}_{5} / \mathrm{SiO}_{2}$ coating using ion beam deposition. Details of the coating process can be found in a recent paper [23]. There were two different coating chambers used to make the samples studied, one large and one small. The primary difference between the chambers is that the ion source in the small chamber is a Kaufman source with tungsten filaments, whereas in the large chamber it is two radio frequency ion sources. In the small chamber, the tungsten filament heats the target as well as the substrate. After coating, each sample was annealed at $600{ }^{\circ} \mathrm{C}$. X-ray examination showed that no large crystals had formed in the coating after annealing.

Each coating consisted of $30 \lambda / 4$ (at $1.064 \mu \mathrm{m}$ ) layers alternating between the two materials, $\mathrm{TiO}_{2}$-doped $\mathrm{Ta}_{2} \mathrm{O}_{5}$ and $\mathrm{SiO}_{2}$. The total thickness was measured in two ways, using reflectivity measurements and with an electron microscope. The methods agreed with each other within $5 \%$, for an average coating thickness of $4.5 \pm 0.1 \mu \mathrm{m}$. One sample was different, a single layer of $\mathrm{TiO}_{2}$-doped $\mathrm{Ta}_{2} \mathrm{O}_{5} 4.7 \mu \mathrm{m}$ thick.

The concentration of $\mathrm{TiO}_{2}$ in $\mathrm{Ta}_{2} \mathrm{O}_{5}$ for each coating was measured in two different ways. First, an estimate was made by comparing the index of refraction of the $\mathrm{TiO}_{2}$-doped $\mathrm{Ta}_{2} \mathrm{O}_{5}$ with pure $\mathrm{Ta}_{2} \mathrm{O}_{5}$ and pure $\mathrm{TiO}_{2}$. A linear relationship was assumed between $\mathrm{TiO}_{2}$ concentration and index so the $\mathrm{TiO}_{2}$ concentration was obtained by interpolation. This is only valid when done between coatings from the same chamber, large or small. A more detailed measurement was made on some samples using electron energy loss spectroscopy, which is described in the appendix. The two methods agreed fairly well when the same coating was studied by both, as seen in table 1. 
Table 1. Concentration of $\mathrm{TiO}_{2}$ in $\mathrm{Ta}_{2} \mathrm{O}_{5}$ as measured by change in index of refraction and by electron energy loss spectroscopy (EELS).

\begin{tabular}{lll}
\hline Coating & {$\left[\mathrm{TiO}_{2}\right]$ Index } & {$\left[\mathrm{TiO}_{2}\right]$ EELS } \\
\hline 0 & $0 \%$ & - \\
1 & $6 \pm 0.6 \%$ & $8.5 \pm 1.2$ \\
2 & $13 \pm 1 \%$ & $20.8 \pm 4.4$ \\
3 & $24 \pm 2 \%$ & $22.5 \pm 2.9$ \\
$4^{\mathrm{a}}$ & $54.5 \pm 5 \%$ & $54 \pm 5$ \\
$5^{\mathrm{a}}$ & $14.5 \pm 1 \%$ & - \\
$6^{\mathrm{b}}$ & $6 \pm 0.6 \%$ & - \\
\hline
\end{tabular}

a Coated in a large coating chamber.

b Single layer of $\mathrm{TiO}_{2}$-doped $\mathrm{Ta}_{2} \mathrm{O}_{5}$.

\subsection{Mechanical loss}

Coated silica discs were used to determine the mechanical loss in the coating. All discs were $7.6 \mathrm{~cm}$ in diameter, with some $2.5 \mathrm{~cm}$ thickness and some $0.25 \mathrm{~cm}$ thickness. The thicker discs were suspended in a wire sling, and had normal mode Q measured with an interferometric readout. The thinner discs were suspended with a welded silica suspension and had modal Q measured using a birefringence readout. Details of the suspension and readout systems for both types of discs can be found in a recent publication [16].

Modal Q were measured on multiple modes of both thin and thick samples for all coated samples. The results are shown in table 2 . The values for the coating loss angles $\phi_{\text {coat, } \|}$ are calculated from the modal Q from

$$
\phi_{\text {coat }, \|}=\left(1 / Q_{\text {coated }}-1 / Q_{\text {uncoated }}\right) /(t \mathrm{~d} U / U),
$$

where $Q$ is the modal Q, measured for the disc both coated and uncoated, $t$ is the thickness of the coating and $\mathrm{d} U / U$ is the ratio of energy stored in the coating per unit coating thickness to the total energy for each given mode shape. These values of $\mathrm{d} U / U$ were calculated using a finite element model [15], using Young's moduli $Y_{\mathrm{Ta} 2 \mathrm{O} 5}=1.4 \times 10^{11} \mathrm{~Pa}$ and $Y_{\mathrm{SiO} 2}=7.2 \times$ $10^{10} \mathrm{~Pa}$, and are shown in table 3 . To determine the coating loss coming from internal friction, the loss predicted from coating thermoelastic damping [19, 20] was subtracted from $\phi$ calculated in equation (9).

The average values for the coating loss for each concentration of $\mathrm{TiO}_{2}$ are shown in table 4. These results, plotted as a function of the percentage of $\mathrm{TiO}_{2}$ cation, are shown in figure 1 .

\subsection{Thermal noise}

A direct, interferometric broadband measurement was made of the thermal noise of the $\mathrm{TiO}_{2}$ doped $\mathrm{Ta}_{2} \mathrm{O}_{5} / \mathrm{SiO}_{2}$ coatings using the small scale interferometer at Caltech. The measurement apparatus and the results for undoped coatings are described elsewhere [18]. Figure 2 shows the result for coated mirrors done in a separate coating run, but using the same coating formula as coating 2 in table 2 except that these mirrors were coated in LMA/Virgo's large coating chamber, while the samples used for the $\mathrm{Q}$ measurements were done in the small chamber. The value for the loss angle obtained by this direct measurement and fit is

$$
\phi_{\text {eff }}=(2.41 \pm 0.15) \times 10^{-6},
$$




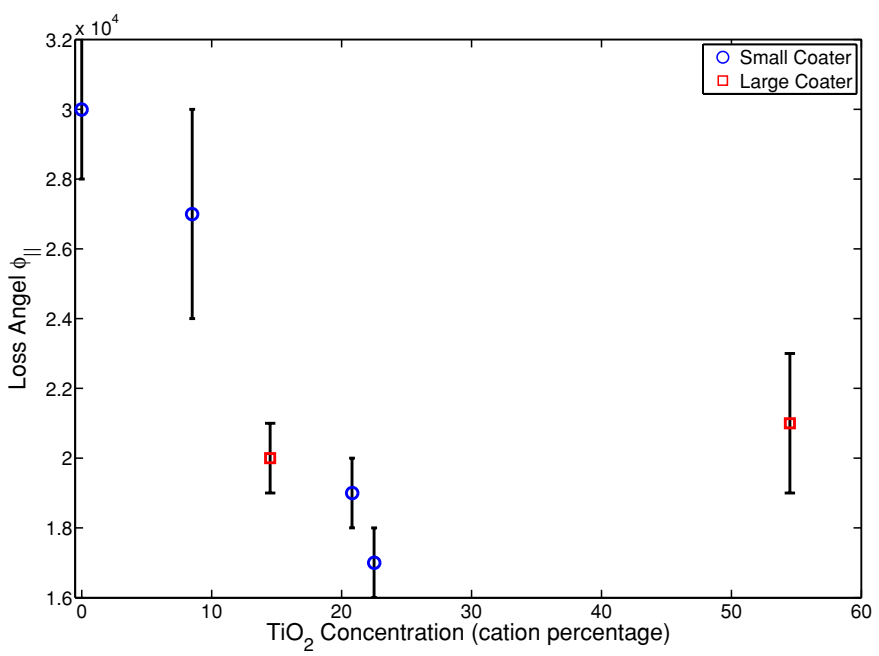

Figure 1. Loss angles with error bars of $\mathrm{TiO}_{2}$-doped $\mathrm{Ta}_{2} \mathrm{O}_{5} / \mathrm{SiO}_{2}$ coating as a function of $\mathrm{TiO}_{2}$ concentration in $\mathrm{Ta}_{2} \mathrm{O}_{5}$. The $\mathrm{TiO}_{2}$ concentration used was the one determined by EELS when available, otherwise the concentration determined by interpolating index changes.

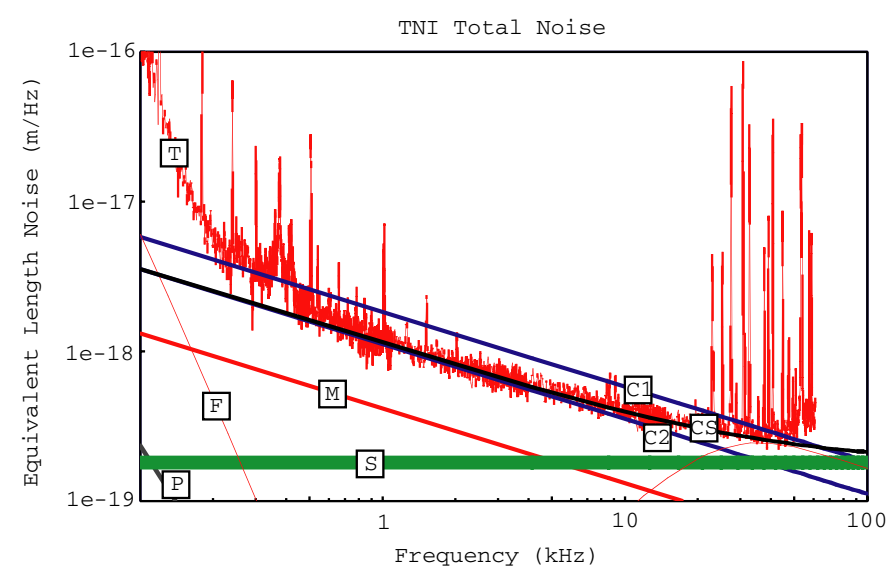

Figure 2. Direct thermal noise measurement of a $\mathrm{TiO}_{2}$-doped $\mathrm{Ta}_{2} \mathrm{O}_{5} / \mathrm{SiO}_{2}$ coating. The curve labelled ' $\mathrm{T}$ ' is the total noise spectrum of the interferometer. ' $\mathrm{C} 1$ ' is the thermal noise of an undoped coating, as previously measured. ' $\mathrm{C} 2$ ' is the thermal noise of the doped coating, where the loss angle was adjusted to fit the data. ' $\mathrm{S}$ ' is the shot noise of the instrument and ' $\mathrm{CS}$ ' is the quadrature sum of this shot noise and the doped-coating thermal noise. ' $\mathrm{M}$ ' is an upper bound on the mirror, or substrate thermal noise, based on in situ measurements of mechanical $\mathrm{Q}$ of the mirrors. ' $\mathrm{F}$ ' is the laser frequency noise and ' $\mathrm{P}$ ' is an upper bound on the pendulum thermal noise.

where $\phi_{\text {eff }}$ is from equation (2). The clear reduction in thermal noise is shown graphically in figure 2 and quantitatively between equations (5) and (10). The result in equation (10) is to be compared with the value predicted from the modal Q results in table 4:

$$
\phi_{\text {eff }}=(4.0 \pm 0.3) \times 10^{-6},
$$

assuming $\phi_{\mathrm{SiO} 2}=1.0 \times 10^{-4}$. The reason for the discrepancy between equations (10) and (11) is not known. 
Table 2. Results of mechanical loss measurements on $\mathrm{TiO}_{2}$-doped $\mathrm{Ta}_{2} \mathrm{O}_{5} / \mathrm{SiO}_{2}$ coatings. All coatings were 30 layers of alternating material with various concentrations of $\mathrm{TiO}_{2}$ in $\mathrm{Ta}_{2} \mathrm{O}_{5}$. Each had an optical thickness $\lambda / 4$ in each layer except for coating 6 . This coating was a single layer of $\mathrm{TiO}_{2}$-doped $\mathrm{Ta}_{2} \mathrm{O}_{5} 4.730 \mu \mathrm{m}$ thick. All coatings were done in the small coating chamber except where noted.

\begin{tabular}{|c|c|c|c|c|}
\hline Coating & Thickness & Frequency & Modal Q & Loss angle $\phi_{\|}$ \\
\hline \multirow[t]{11}{*}{0} & \multirow[t]{3}{*}{ Thin } & 2733 & $5.4 \times 10^{5}$ & $2.5 \times 10^{-4}$ \\
\hline & & 2735 & $5.3 \times 10^{5}$ & $2.5 \times 10^{-4}$ \\
\hline & & 4130 & $4.3 \times 10^{5}$ & $2.8 \times 10^{-4}$ \\
\hline & \multirow[t]{8}{*}{ Thick } & 20180 & $4.8 \times 10^{6}$ & $2.2 \times 10^{-4}$ \\
\hline & & 20183 & $3.6 \times 10^{6}$ & $3.2 \times 10^{-4}$ \\
\hline & & 28383 & $3.0 \times 10^{6}$ & $3.3 \times 10^{-4}$ \\
\hline & & 28387 & $3.3 \times 10^{6}$ & $2.9 \times 10^{-4}$ \\
\hline & & 47349 & $5.6 \times 10^{6}$ & $3.7 \times 10^{-4}$ \\
\hline & & 47363 & $6.3 \times 10^{6}$ & $2.9 \times 10^{-4}$ \\
\hline & & 73454 & $3.0 \times 10^{6}$ & $4.0 \times 10^{-4}$ \\
\hline & & 73458 & $3.8 \times 10^{6}$ & $2.7 \times 10^{-4}$ \\
\hline \multirow[t]{9}{*}{1} & \multirow[t]{5}{*}{ Thin } & 2653 & $7.6 \times 10^{5}$ & $1.8 \times 10^{-4}$ \\
\hline & & 2666 & $2.0 \times 10^{5}$ & $7.1 \times 10^{-4}$ \\
\hline & & 4026 & $3.6 \times 10^{5}$ & $3.6 \times 10^{-4}$ \\
\hline & & 6045 & $7.4 \times 10^{5}$ & $1.9 \times 10^{-4}$ \\
\hline & & 6078 & $7.6 \times 10^{5}$ & $1.8 \times 10^{-4}$ \\
\hline & \multirow[t]{4}{*}{ Thick } & 20191 & $4.9 \times 10^{6}$ & $2.2 \times 10^{-4}$ \\
\hline & & 28428 & $3.5 \times 10^{6}$ & $2.6 \times 10^{-4}$ \\
\hline & & 47423 & $5.4 \times 10^{6}$ & $4.1 \times 10^{-4}$ \\
\hline & & 73515 & $3.4 \times 10^{6}$ & $3.4 \times 10^{-4}$ \\
\hline \multirow[t]{11}{*}{2} & \multirow[t]{7}{*}{ Thin } & 2706 & $8.9 \times 10^{5}$ & $1.5 \times 10^{-4}$ \\
\hline & & 2711 & $8.8 \times 10^{5}$ & $1.5 \times 10^{-4}$ \\
\hline & & 4101 & $6.8 \times 10^{5}$ & $1.7 \times 10^{-4}$ \\
\hline & & 6165 & $8.4 \times 10^{5}$ & $1.6 \times 10^{-4}$ \\
\hline & & 6184 & $8.6 \times 10^{5}$ & $1.6 \times 10^{-4}$ \\
\hline & & 9464 & $6.3 \times 10^{5}$ & $2.0 \times 10^{-4}$ \\
\hline & & 9465 & $6.1 \times 10^{5}$ & $2.0 \times 10^{-4}$ \\
\hline & \multirow[t]{4}{*}{ Thick } & 20239 & $6.7 \times 10^{6}$ & $1.9 \times 10^{-4}$ \\
\hline & & 28488 & $5.4 \times 10^{6}$ & $1.8 \times 10^{-4}$ \\
\hline & & 47466 & $10.0 \times 10^{6}$ & $2.5 \times 10^{-4}$ \\
\hline & & 73599 & $5.2 \times 10^{6}$ & $2.5 \times 10^{-4}$ \\
\hline \multirow[t]{9}{*}{3} & \multirow[t]{5}{*}{ Thin } & 2722 & $9.3 \times 10^{5}$ & $1.5 \times 10^{-4}$ \\
\hline & & 4111 & $6.4 \times 10^{5}$ & $1.9 \times 10^{-4}$ \\
\hline & & 6197 & $9.1 \times 10^{5}$ & $1.5 \times 10^{-4}$ \\
\hline & & 9517 & $6.4 \times 10^{5}$ & $2.0 \times 10^{-4}$ \\
\hline & & 9519 & $6.6 \times 10^{5}$ & $1.9 \times 10^{-4}$ \\
\hline & \multirow[t]{4}{*}{ Thick } & 20245 & $7.0 \times 10^{6}$ & $1.7 \times 10^{-4}$ \\
\hline & & 28500 & $6.3 \times 10^{6}$ & $1.4 \times 10^{-4}$ \\
\hline & & 47485 & $11.3 \times 10^{6}$ & $2.0 \times 10^{-4}$ \\
\hline & & 73620 & $7.0 \times 10^{6}$ & $1.5 \times 10^{-4}$ \\
\hline \multirow[t]{5}{*}{$4^{\mathrm{a}}$} & \multirow[t]{5}{*}{ Thin } & 2723 & $9.2 \times 10^{5}$ & $1.5 \times 10^{-4}$ \\
\hline & & 2724 & $9.7 \times 10^{5}$ & $1.4 \times 10^{-4}$ \\
\hline & & 4114 & $6.0 \times 10^{5}$ & $2.0 \times 10^{-4}$ \\
\hline & & 6200 & $8.1 \times 10^{5}$ & $1.7 \times 10^{-4}$ \\
\hline & & 9524 & $6.1 \times 10^{5}$ & $2.1 \times 10^{-4}$ \\
\hline
\end{tabular}




\begin{tabular}{|c|c|c|c|c|}
\hline Coating & Thickness & Frequency & Modal Q & Loss angle $\phi_{\|}$ \\
\hline & Thick & 20241 & $5.4 \times 10^{6}$ & $2.5 \times 10^{-4}$ \\
\hline & & 28493 & $4.1 \times 10^{6}$ & $2.7 \times 10^{-4}$ \\
\hline & & 47467 & $6.1 \times 10^{6}$ & $5.3 \times 10^{-4}$ \\
\hline & & 73598 & $5.0 \times 10^{6}$ & $2.7 \times 10^{-4}$ \\
\hline \multirow[t]{4}{*}{$5^{a}$} & Thick & 20193 & $6.5 \times 10^{6}$ & $2.0 \times 10^{-4}$ \\
\hline & & 28400 & $5.4 \times 10^{6}$ & $1.8 \times 10^{-4}$ \\
\hline & & 47398 & $10.9 \times 10^{6}$ & $2.2 \times 10^{-4}$ \\
\hline & & 73524 & $6.2 \times 10^{6}$ & $1.9 \times 10^{-4}$ \\
\hline \multirow[t]{4}{*}{$6^{\mathrm{b}}$} & Thick & 20226 & $2.9 \times 10^{6}$ & $2.6 \times 10^{-4}$ \\
\hline & & 28462 & $1.9 \times 10^{6}$ & $3.7 \times 10^{-4}$ \\
\hline & & 47430 & $3.6 \times 10^{6}$ & $4.4 \times 10^{-4}$ \\
\hline & & 73588 & $2.4 \times 10^{6}$ & $3.3 \times 10^{-4}$ \\
\hline
\end{tabular}

a Coated in large coating chamber.

${ }^{\text {b }}$ Single layer of $\mathrm{TiO}_{2}$-doped $\mathrm{Ta}_{2} \mathrm{O}_{5}$.

Table 3. Ratio of energy in the coating (per unit coating thickness) to total energy for modes of the thin and thick samples.

\begin{tabular}{lcc}
\hline Thickness & $\begin{array}{l}\text { Approximate modal } \\
\text { frequency }(\mathrm{Hz})\end{array}$ & $\mathrm{d} U / U(1 / \mathrm{m})$ \\
\hline Thin & 2700 & 1584 \\
& 4100 & 1659 \\
& 6200 & 1581 \\
Thick & 9500 & 1624 \\
& 20200 & 142.4 \\
& 28500 & 153.2 \\
& 47400 & 52.33 \\
& 73500 & 109.1 \\
\hline
\end{tabular}

Table 4. Coating mechanical loss of $\mathrm{TiO}_{2}$-doped $\mathrm{Ta}_{2} \mathrm{O}_{5} / \mathrm{SiO}_{2}$ coatings. All coatings were done in the small coating chamber except where noted.

\begin{tabular}{ll}
\hline Coating & $\phi_{\text {coat, }, \|}\left(\times 10^{-4}\right)$ \\
\hline 0 & $3.0 \pm 0.2$ \\
1 & $2.7 \pm 0.3$ \\
2 & $1.9 \pm 0.1$ \\
3 & $1.7 \pm 0.1$ \\
$4^{\mathrm{a}}$ & $2.1 \pm 0.2$ \\
$5^{\mathrm{a}}$ & $2.0 \pm 0.1$ \\
$6^{\mathrm{b}}$ & $3.5 \pm 0.4$ \\
\hline
\end{tabular}

a Coated in large coating chamber.

b Single layer of $\mathrm{TiO}_{2}$-doped $\mathrm{Ta}_{2} \mathrm{O}_{5}$.

\subsection{Optical absorption}

Optical absorption was measured using photothermal, common-path interferometry (PCI), which is a modified thermal lensing technique that exploits the thermo-optic effect (index 


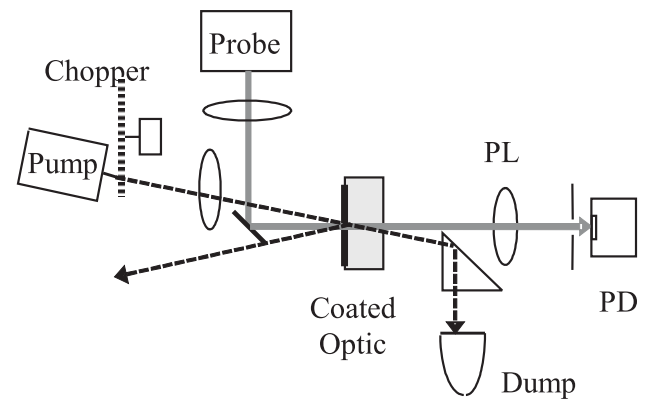

Figure 3. Crossed-beam setup for low absorption photothermal, common-path interferometry measurement. PL: projecting lens, PD: photodetector.

Table 5. Optical absorption of $\mathrm{TiO}_{2}$-doped $\mathrm{Ta}_{2} \mathrm{O}_{5} / \mathrm{SiO}_{2}$ coatings and indices of refraction of individual $\mathrm{TiO}_{2}$-doped $\mathrm{Ta}_{2} \mathrm{O}_{5}$ layers within those coatings. Index of refraction comparisons is only valid between coatings from the same coating chamber.

\begin{tabular}{lll}
\hline Coating & Index $n$ & Absorption $(\mathrm{ppm})$ \\
\hline 0 & $2.065 \pm 0.005$ & $0.9 \pm 0.2$ \\
1 & $2.075 \pm 0.005$ & $1.1 \pm 0.1$ \\
2 & $2.092 \pm 0.005$ & $1.0 \pm 0.1$ \\
3 & $2.119 \pm 0.005$ & $1.1 \pm 0.1$ \\
$4^{\mathrm{a}}$ & $2.180 \pm 0.005$ & $2.5 \pm 0.5$ \\
$5^{\mathrm{a}}$ & $2.070 \pm 0.005$ & $0.9 \pm 0.1$ \\
$6^{\mathrm{b}}$ & $2.075 \pm 0.005$ & $4.5 \pm 0.5$ \\
\hline
\end{tabular}

a Coated in a large coating chamber.

b Single layer of $\mathrm{TiO}_{2}$-doped $\mathrm{Ta}_{2} \mathrm{O}_{5}$.

of refraction dependence on temperature: $\mathrm{d} n / \mathrm{d} T)$. It differs from standard far-field thermal lensing by utilizing a near-field detection scheme which approaches in sensitivity that of interferometric absorption measurement methods. Phase distortions, $\delta \phi$, of the probe beam due to heating by an intersecting pump beam in a skewed cylindrical region (approximately $75 \mu \mathrm{m}$ diameter by $500 \mu \mathrm{m}$ long) are transformed into perturbations of the probe beam intensity, $\Delta I / I \approx \Delta \phi$, that are easily detectable using a lock-in detection technique which gives both amplitude and phase. For materials with $\mathrm{d} n / \mathrm{d} T$ around $10^{-5} / \mathrm{K}$ and with pump powers of $1 \mathrm{~W}$, resolutions of $\leqslant 1 \mathrm{ppm}$ in terms of the absorbed fraction of pump power are readily achievable. The signal phase can be used to discriminate between probe light scattered from surface imperfections compared to that diffracted by the thermal wave emanating from the heated surface.

The PCI technique used here utilizes a chopped pump beam at $1064 \mathrm{~nm}$ which is crossed with a wider $632.8 \mathrm{~nm}$ probe beam inside the sample, see figure 3. In the case of high reflectivity (HR) coatings, virtually all the pump beam is reflected. As long as substrate losses are negligible as is the case with fused silica, the heat deposited in the coating due to optical absorption of the pump wavelength is the only source that heats the underlying substrate (by thermal conduction). In order for the probe beam to sense the change in the local optical index via the resulting phase distortions, it is necessary that the multi-layer dielectric HR coatings be transparent to the probe beam, as are the coatings studied here. Results of optical absorption measurements as well as index of refraction results are shown in table 5. 


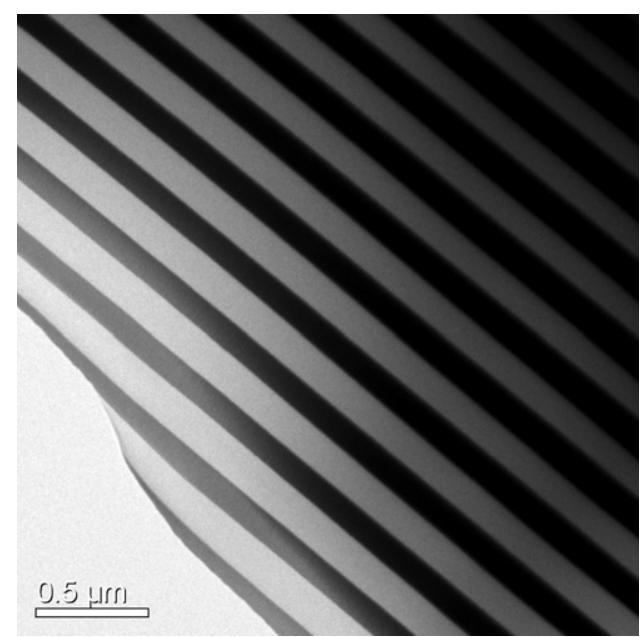

Figure 4. Bright field TEM image of the multi-layer structure: the lighter layers are the amorphous $\mathrm{SiO}_{2}$ layers and the darker layers are the $\mathrm{Ta}_{2} \mathrm{O}_{5}-\mathrm{TiO}_{2}$ layers.

In these experiments, a commercial neutral density (ND) filter consisting of a partially transparent metallic film on a low-loss fused silica substrate is used as a coating optical absorption standard. In the PCI technique, the detected signal amplitude is linear with respect to pump beam intensity and, therefore, straightforward linear scaling can be used to relate the signal levels from unknowns to those of the easily measured ND filters. With both coated standards and unknowns, the coated surfaces are positioned near the centre of the sampling volume where maximum signal is achieved. With $10 \mathrm{~W}$ of pump power, resolutions of $\leqslant 0.1 \mathrm{ppm}$ are achievable.

\section{Conclusion}

The mechanical loss results in table 4 and figure 1 show that adding $\mathrm{TiO}_{2}$ to $\mathrm{Ta}_{2} \mathrm{O}_{5}$ reduces the mechanical loss. Differing concentrations of $\mathrm{TiO}_{2}$ do not affect the loss nearly as much as simply the presence or absence of $\mathrm{TiO}_{2}$. This reduction of nearly half in the loss angle of the coating corresponds to a significant improvement in thermal noise, which translates into greater astronomical reach for advanced interferometers. The optical absorption seen in table 5 would be problematic in an advanced interferometer, but slight improvements in coating technique may be able to bring these numbers down to acceptable levels. The addition of $\mathrm{TiO}_{2}$ does appear to increase the optical absorption slightly, so using minimal concentrations will be useful. Changes in annealing cycles are known to affect optical absorption, as does levels of contamination, so both of these variables could potentially be improved as well. Further measurements on the inhomogeneity of the optical loss, the scatter and the reproducibility of all properties will be necessary before $\mathrm{TiO}_{2}$-doped $\mathrm{Ta}_{2} \mathrm{O}_{5} / \mathrm{SiO}_{2}$ could be accepted for use in an actual gravitational-wave interferometer.

\section{Acknowledgments}

The LIGO Observatories were constructed by the California Institute of Technology and Massachusetts Institute of Technology with funding from the National Science Foundation 
under cooperative agreement PHY-9210038. The LIGO Laboratory operates under cooperative agreement PHY-0107417. We thank Zhigang Pan and Slawomir Gras for checking the thermal noise formulae. We also gratefully acknowledge funding from NSF grant PHY-0355118(HWS). This paper has been assigned LIGO Document Number LIGOP050048-00-R.

\section{Appendix. Measurement of the composition of the $\mathrm{Ta}_{2} \mathrm{O}_{5}-\mathrm{TiO}_{2}$ layers}

The measurement of $\mathrm{Ti}$ dopant concentration was performed using electron energy loss spectroscopy (EELS) [24] on a FEI Tecnai T20 transmission electron microscope (TEM) operated at $200 \mathrm{kV}$ equipped with a Gatan image filter (GIF), see figure 4. The edges in the EELS spectrum used for quantification were the $\mathrm{O}-\mathrm{K}$ edge at $532 \mathrm{eV}$, the Ti- $\mathrm{L}_{2.3}$ pair starting at $456 \mathrm{eV}$ and the Ta $\mathrm{N}_{4,5}$ edge at $229 \mathrm{eV}$ (details of the different atomic transitions used in EELS and their nomenclature may be found in a recent book [24]). The O-K and Ti-L edges arise from transitions between relatively simple inner atomic shells, and Hartree-Slater calculations of the partial cross sections are readily available and reliable. In contrast to this, $\mathrm{N}$ edges correspond to transitions among the higher atomic shells and are thus rather more complex; consequently, no analytical representation of the partial cross section for the $\mathrm{Ta}_{4,5}$ edge was available. This problem was circumvented by determining the partial cross section experimentally from 20 spectra from the Ta2O5 layers in the undoped sample 0 , assuming that the Ta:O ratio was the stoichiometric 2:5. This was then used with the calculated Ti partial cross section to quantify spectra recorded for all four doped samples.

Quantification of the doped $\mathrm{Ta}_{2} \mathrm{O}_{5}$ layers in coating 1 was carried out using 20 spectra collected from different thin regions $(<50 \mathrm{~nm})$ and gave a result of $8.5 \pm 1.2$ cation percentage of $\mathrm{Ti}$ (with the balance Ta). For sample 2, a similar measurement was carried out giving a result of $20.8 \pm 4.4$ cation percentage of Ti. Only four spectra could be collected from coating 3 due to much of the sample being too thick for accurate EELS analysis; quantification yielded a result of $22.5 \pm 2.9$ cation percentage of Ti. For coating 4, 20 spectra were recorded, but more difficulties were encountered with the analysis. Accurate quantification relies on correct subtraction of the background under the edge, but this can become difficult when the edge is only a small feature above the background. The Ta edge is fairly weak and has a delayed onset and background subtraction was a problem in coating 4 . Different background models were tried, some which subtracted too much, and others which subtracted too little. The best-fit background model led to a consistent quantification of the 20 spectra to give a $54 \pm 5$ cation percentage of $\mathrm{Ti}$, although it is believed that too much background was removed here. An alternative background model which did not remove enough led to quantifications in the low 40s (cation percentage of Ti). It seems likely that the real figure is about 5 cation percentage lower than the above quoted figure and is of the order of 50 cation percentage Ti.

\section{References}

[1] Abbott B et al 2004 Detector description and performance for the first coincidence observations between LIGO and GEO Nucl. Instrum Methods Phys. Res. A 517/1-3 154-79

[2] Acernese F et al 2002 Tatus of VIRGO Class. Quantum Grav. 191421

[3] Ando M (and the TAMA collaboration) 2002 Current status of TAMA Class. Quantum Grav. 191409

[4] Fritschel P 2003 Second generation instruments for the laser interferometer gravitational wave observatory Gravitational-Wave Detection ed M Cruise and P Saulson (Proc. SPIE vol 4856) pp 282-91

[5] Thorne K S and Cutler C 2002 An overview of gravitational wave sources Proc. 16th Int. Conf. on General Relativity and Gravitation ed N T Bishop and S D Maharaj (Singapore: World Scientific) pp 72-112 
[6] Harry G M et al 2002 Thermal noise in interferometric gravitational wave detectors due to dielectric optical coatings Class. Quantum Grav. 19 897-917

[7] Numata K, Kemery A and Camp J 2004 Phys. Rev. Lett. 93250602

[8] Callen H B and Greene R F 1952 On a theorem of irreversible thermodynamics Phys. Rev. 86 702-10

[9] Levin Yu 1998 Internal thermal noise in the LIGO test masses: a direct approach Phys. Rev. D 57 659-63

[10] Lawrence R, Ottaway D, Fritschel P and Zucker M 2004 Active correction of beam heating induced phase distortions in optics via external radiative thermal acutation Opt. Lett. $292635-7$

[11] Harry G M et al 2004 Photonic applications in astronomy, biomedicine, imaging, materials processing, and education Proc. SPIE: 5578 (Bellingham, WA: SPIE)

[12] Yamamoto K et al 2004 Mechanical loss of the reflective coating and fluorite at low temperature Class. Quantum Grav. 21-5 S1075-81

[13] Yamamoto K, Ando M, Kawabe K and Tsubono K 2002 Thermal noise caused by an inhomogeneous loss in the mirrors used in the gravitational wave detector Phys. Lett. A 305 18-25

[14] Beauville F (for The VIRGO Collaboration) 2004 The VIRGO large mirrors: a challenge for low loss coatings Class. Quantum Grav. 21 S935-45

[15] Crooks D R M et al 2002 Excess mechanical loss associated with dielectric mirror coatings on test masses in interferometric gravitational wave detectors Class. Quantum Grav. 19-5 883-96

[16] Penn S D et al 2003 Mechanical loss in tantala/silica dielectric mirror coatings Class. Quantum Grav. $202917-28$

[17] Crooks D R M et al 2006 Experimental measurements of mechanical dissipation associated with dielectric coatings formed using $\mathrm{SiO}_{2}, \mathrm{Ta}_{2} \mathrm{O}_{5}$, and $\mathrm{Al}_{2} \mathrm{O}_{3}$ Class. Quantum Grav. 23 4953-65

[18] Black E D et al 2004 Direct observation of broadband coating thermal noise in a suspended interferometer $P$ hys. Lett. A 328 1-5

[19] Fejer M M, Rowan S, Cagnoli G, Crooks D R M, Gretarsson A, Harry G M, Hough J, Penn S D, Sneddon P H and Vyatchanin S P 2004 Thermoelastic dissipation in inhomogeneous media: loss measurements and displacement noise in coated test masses for interferometric gravitational wave detectors Phys. Res. D 70 082003

[20] Braginsky V B and Vyatchanin S P 2003 Thermodynamical fluctuations in optical mirror coatings Phys. Lett. A 312/3-4 224-55

[21] Numata K, Ando M, Yamamoto K, Otsuka S and Tsubono K 2003 Wide-band direct measurement of thermal fluctuations in an interferometer Phys. Rev. Lett. 91260602

[22] Cimma B et al 2004 Original optical metrologies of large componants Proc. SPIE: 5252 (Bellingham, WA: SPIE)

[23] Mackowski J M, Morgado N, Pinard L and Remillieux A 2004 Low loss coatings for the Virgo large mirrors Proc. SPIE: 5250 (Bellingham, WA: SPIE)

[24] Egerton R F 1996 Electron Energy-Loss Spectroscopy in the Electron Microscope 2nd edn (New York: Plenum) 